\title{
Analysis of measured data and its effect on active sonar working depth in the Yellow Sea*
}

\author{
Zhan $\mathrm{He}$ \\ 1. Naval Aeronautical Engineering College \\ Yantai, China \\ 2. Unit 92074 \\ Ningbo, China
}

\author{
Yang Rijie \\ Naval Aeronautical Engineering College \\ Yantai, China
}

\begin{abstract}
Based on the WOD13 data of the Yellow Sea, we analyze the sound speed profile and sound transmission loss of some position in it, and summarize its variation regulation. Utilizing the Bellhop model, we calculate the detect range of active sonar at different working depths, pick up the optimal working depth at which can make active sonar achieve the farthest detect range, and offer the essential theoretical research to utilize ocean data in this area and to analyze or predict active sonar performance.
\end{abstract}

Keywords-Yellow Sea, active sonar, optimal working depth

\section{INTRODUCTION}

The yellow sea lies between China and the Korean peninsula, it is China's important Marine strategic position and has important military value and research significance. The average depth of this area is shallow, the deepest position is 140 meters located in the north of Jeju Island. And its depth from the south to the north gradually becomes more and more shallow. The Yellow Sea has myriad coastal current and is influenced by several cold or warm currents in different seasons, making its acoustic environment very complex[1-3]. Therefore, dealing with the measured data of Yellow Sea, analyzing its change law, providing theoretical basis of active sonar performance prediction and working depth selection for anti-submarine warfare, has important research significance.

\section{ANALYSIS OF THE VARIATION OF SOUND SPEED IN THE YELLOW SEA}

\section{A. Data sources and formats}

The original data used is the WOD13 data[4] from NOAA(http://www.nodc.noaa.gov). Sound speed value at extended standard depths is calculated by emprical formula[5] with temperature, salinity and pressure of original data. The whole sound speed profile from sea surface to bottom is calculated by cubic spline interpolation[6,7] which could make the profile smooth and close to real value.

\section{B. Analysis of variation of sound speed profile}

Choose a point, named point $\mathrm{A}$ and located at $125^{\circ} \mathrm{E}$ and $34.5^{\circ} \mathrm{N}$, in the south of the Yellow Sea. Take February, May, August and November as the representative month of four

Funded by by Chinese national natural science foundation (61271444). seasons separately. So the SSP, sound speed profile, of four seasons at point $\mathrm{A}$ is shown in figure 1 .

Fig. 1. SSP of four seasons at point A.

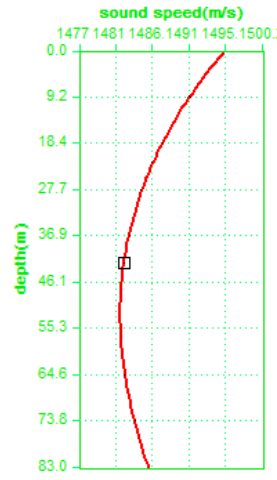

(a)spring

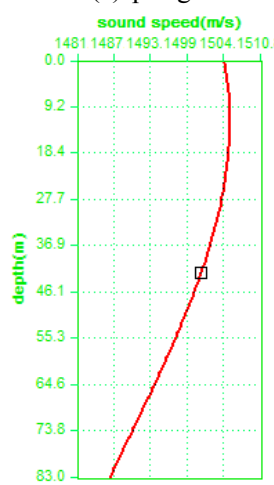

(c)autumn

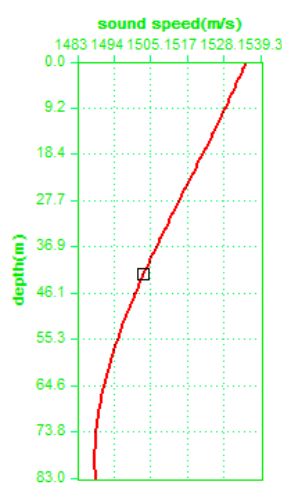

(b)summer

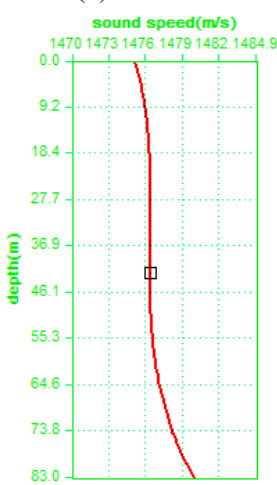

(d)winter
According to Fig. 1, at point A, there are obvious differences in sound speed profile while season changes, and the differences are embodied in the entire water layer from sea surface to bottom.

In spring, the coastal water and the outer sea water entering from continental shelf are mixed to form mixed water mass. By rising temperature and lowering salinity, this water mass splits into upper water mass with higher temperature and lower water mass with higher salinity. This phenomenon makes the SSP be that shown in Fig. 1(a). 
In summer, the temperature at sea surface rises up sharply. At the same time, the bottom cold water mass emerging in spring changes into cyclonic circulation, which makes the temperature at bottom keep low. So the SSP in summer is a gradient one with larger negative value, as shown in Fig. 1(b).

In autumn, the temperature at sea surface is lower than that in summer, and the sound speed is also lower. But the change in temperature and salinity at bottom is not obvious. So the sound speed gradient is still negative, however, the absolute value of gradient becomes lower and lower.

In winter, influenced by the Tsushima warm current which brings water with higher temperature and higher salinity, mixed water mass starts to appear, sound speed at bottom becomes higher and higher. Meanwhile, influenced by cold air, sound speed at sea surface is getting lower and lower. From 25 to 60 meters, it is the isothermal layer.

\section{ANALYSIS OF SOUND TRANSMISSION LOSS AND ACTIVE SONAR WORKING DEPTH IN THE YELLOW SEA}

\section{A. Analysis of sound transmission loss}

Sonar detect range prediction would be used to analyze and evaluate the effect of detection, and its detect range is determined by sound transmission loss. So, based on the SSP data, it is necessary to analyze the transmission loss in the Yellow Sea.

Suppose that active sonar is placed at point A, seabed terrain is calculated with the Etopo data[8] from U.S. National Geophysical Data Center, sound propagation is simulated with the BELLHOP model[9-11], active sonar works at 50 meters depth, sound frequency is $10 \mathrm{kHz}$, sound beam angle is between -15 and 15 degrees, seafloor sediment is mixture of sand and mud whose density is $1550 \mathrm{~kg} / \mathrm{m} 3$, sound speed at sea bottom is $1600 \mathrm{~m} / \mathrm{s}$, attenuation coeffcient is $0.2 \mathrm{~dB} / \lambda$. According to SSP in Fig. 1, the sound transmission loss from point A to $20 \mathrm{~km}$ east is shown in Fig. 2.

Fig. 2. TL in four seasons at point A.

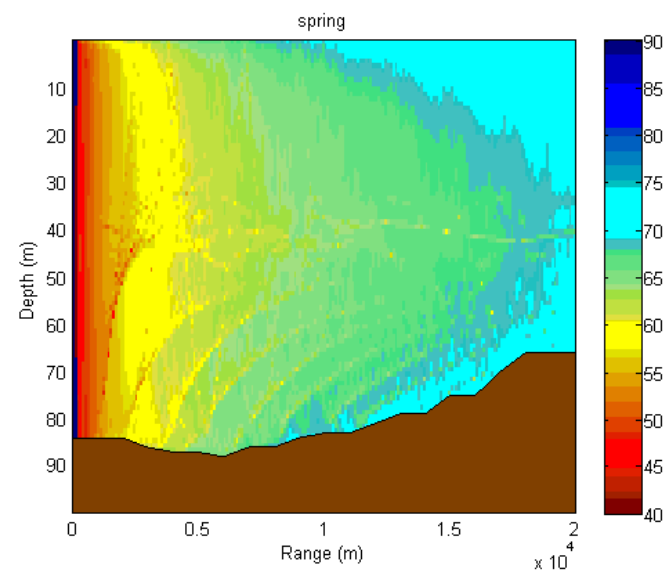

(a)Spring

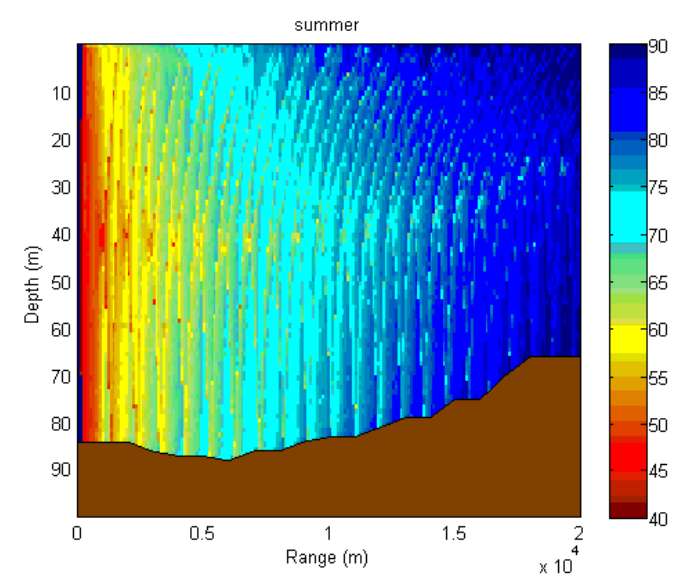

(b)Summer

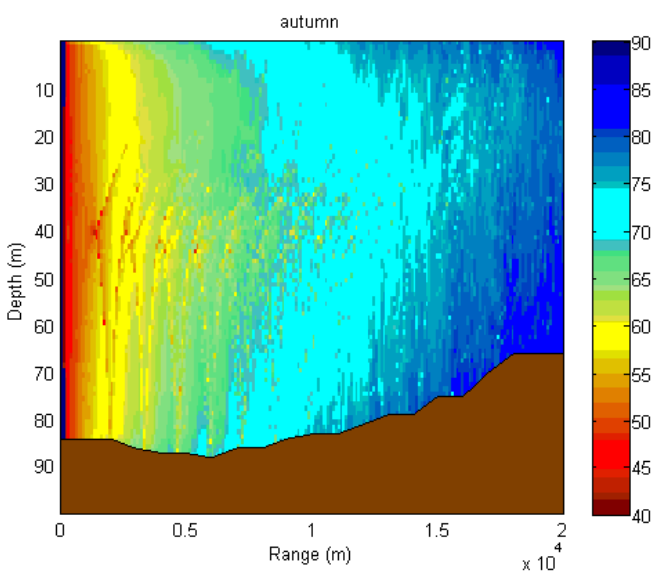

(c)autumn

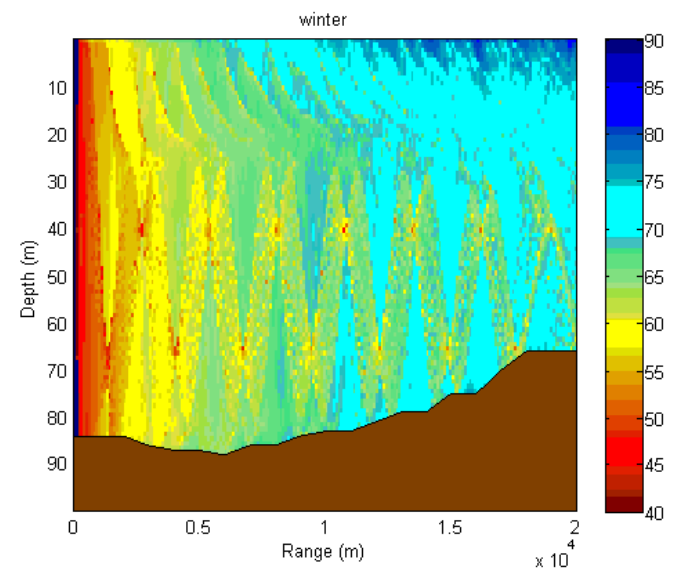

(d)winter

According to Fig. 2, within 7 kilometers, where the terrain change is not obvious, there is no shadow zone in all four seasons. The transmission loss at every depth is restrained within $70 \mathrm{~dB}$, even $60 \mathrm{~dB}$ in spring. But beyond 7 kilometers, the bend effect caused by sound speed gradient becomes obvious, different features appear in different seasons.

In spring, the minimum sound speed value is at about 50 meters depth. When sound source works at 40 meters, sound energy could be focused around this depth, reflect loss at sea surface and bottom could be reduced effectively, sound propagation is very ideal. Within 13 kilometers, transmission 
loss at most depths is smaller than $68 \mathrm{~dB}$. When decaying to 68 $\mathrm{dB}$ at 40 meters depth, the transmission range can even reach 16 kilometers.

Summer has the worst propagation results, shadow zone appears at about 7 kilometers. But due to the large value of sound speed gradient, sound rays are reflected by surface and bottom so frequently that the scale of shadow zone is not large. From the energy point of view, transmission loss is smaller than $64 \mathrm{~dB}$ at every depths within 4 kilometers, while bigger than $68 \mathrm{~dB}$ at most depths beyond 6 kilometers. We can imagine how fastly the energy decays.

In autumn, although its sound speed profile is also of negative gradient, largest value of which is about $-0.38 \mathrm{~m} / \mathrm{s}$, only half of that in summer, the reflect effect is not as obvious as that in summer. Within 12 kilometers, at each distance, the transmission loss value at each depth is almost the same. In other words, no matter how does underwater target change its depth, the detect effect of active sonar would remain stable. Beyond 12 kilometers, energy concentrate toward sea surface as the terrain uplift.

In winter, in conditions of positive-zero-positive gradient, taking isothermal layer' s starting depth which is about 25 meters as dividing line, there are obvious differences in propagation rules. The upper layer embodies the characteristics of propagation in positive gradient profile while the lower layer embodies the characteristics of propagation in isothermal layer. This makes energy scatter widely, and about 10 meters thick area in which transmission loss is larger than $70 \mathrm{~dB}$ appear in upper layer around sea surface. In the lower layer, the area with larger than $70 \mathrm{~dB}$ transmission loss appears at a distance of 10.5 kilometers, its span length is about 2 kilometers. The span length increases with the increase of distance.

\section{B. Analysis of active sonar working depth}

Not only the acoustic environment but also working depth affect detect range of active sonar. In conditions of acoustic environment analyzed above, suppose that underwater target is at 30 meters depth, the FOM of sonar is $80 \mathrm{~dB}$, detection probability is $75 \%$. When active sonar works at depth from 15 to 70 meters with an interval of 5 meters, its detect range variation is shown in Fig. 3.

Fig. 3. Detect range at different working depth

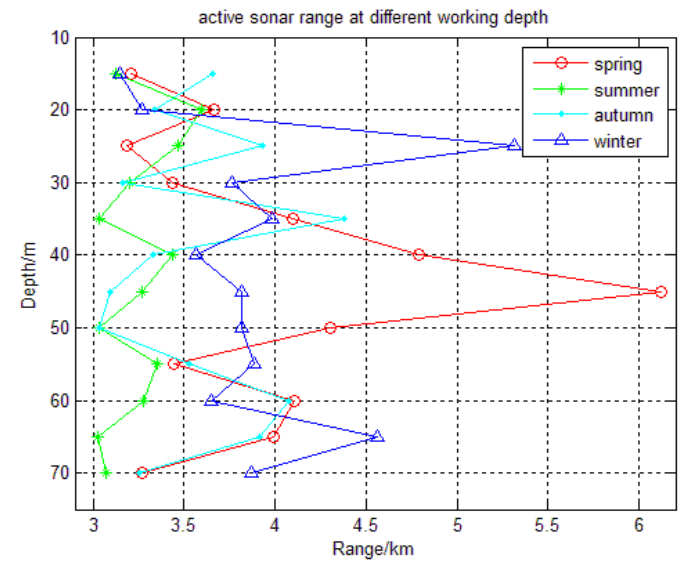

According to Fig. 3, although target is at the same depth, there are still large differences in detect range with different working depth. In spring, the maximum range is 6.12 kilometers at working depth of 45 meters while the minimum range is 3.19 kilometers at working depth of 25 meters. In summer, the maximum range is 3.59 kilometers at 20 meters while the minimum range is 3.03 kilometers at 65 meters. In autumn, the maximum range is 4.38 kilometers at 35 meters while the minimum range is 3.03 kilometers at 50 meters. In winter, the maximum range is 5.32 kilometers at 25 meters while the minimum range is 3.03 kilometers at 65 meters.

In spring, there is a turning point from negative to positive gradient at 46 meters depth, most energy is concentrated around this depth. Among all 12 depths simulated, the farthest 4 detect ranges are distributed around this depth. Detect range at 45 meters working depth is also the farthest in 4 seasons. So, in spring, it is recommended setting working depth at the speed turning depth or half of sea depth.

In summer, detect ranges at different depths are close and overall near, the farthest is only 3.59 kilometers. This is obviously due to sharp negative gradient sound speed profile. So, in summer, it is recommended setting working depth at one forth of sea depth to decrease energy decay cause by frequently bottom reflect.

In autumn, detect ranges at working depth below 55 meters are almost the same as them in spring. This is due to their similar transmission loss distribution within 5 kilometers as shown in Fig. 2. But there is no turning point of speed gradient in autumn, meanwhile its energy concentrate toward sea surface, therefore, at depth of 35 meters it achieves its farthest detect range. So, in autumn, it is recommended setting working depth at three-eighths of sea depth.

In winter, when working depth is set at 25 meters depth, at which the sound speed gradient changes from positive to zero, energy concentrate around this depth so greatly that it achieves the second farthest detect range in all 4 seasons. In addition, when working depth is set at 65 meters depth, at which the sound speed gradient changes from zero to positive, the detect range is also farther. This is due to the positive gradient profile close to bottom bends sound rays upward to decrease the times of bottom reflect. In isothermal layer, from 30 to 60 meters, detect ranges at different depth are close, the difference between the maximum and minimum values is less than 0.5 kilometers. So, in winter, it is recommended setting working depth at one forth or three-fourths of sea depth.

\section{CONCLUSION}

The acoustic environment of the Yellow Sea is very complex. In different seasons its sound speed profile and transmission loss vary greatly, this has great influence on detecting underwater target. We chose a point in this area and took its WOD13 data as the original data. Based on the data we analyzed its sound speed profile and transmission loss variation rules in different seasons, calculated detect range of active sonar at different working depth with the BELLHOP model, analyzed the differences in detect range and cause of these differences, summarized the optimal working depth in all seasons for active sonar in the sense of maximum detect range, 
provided certain theoretical support for utilizing acoustic environment and predicting sonar performance in this area.

\section{REFERENCES}

[1] QIAO Lulu, etc. , "Winter heat budget in the Huanghai Sea and the effect from Huanghai Warm Current(Yellow Sea Warm Current)", Acta Oceanol. Sin., Beijing, Vol. 30, No. 5, pp. 56-63, 2011.

[2] LIU Ping, etc. , "Seasonal variability of the zooplankton community in the southwest of the Huanghai Sea(Yellow Sea) Cold Water Mass", Acta Oceanol. Sin., Beijing, Vol. 31, No. 4, pp. 127-139, 2012.

[3] WANG Bin, etc. , "Effects of topography on the sub-tidal circulation in the southwestern Huanghai Sea(Yellow Sea) in summer", Acta Oceanol. Sin., Beijing, Vol. 32, No. 3, pp. 1-9, 2013.

[4] http://www.nodc.noaa.gov.

[5] Urick, R.J., Principles of Underwater Sound, 3rd ed., McGraw-Hill Inc.: Los Altos, CA, USA, 1983.
[6] Chen Wenlue, Wang Ziyang, "The application of cubic splines interpolation in the project fitting", Journal of Central China Normal University(Nat. Sci.), Wuhan, Vol. 38, No. 4, pp. 418-422, 2004.

[7] Peng Rongli, Jin Ping, Ouyang Jianguo, "Data fitting and application of listing functions to engineering", Engineering Journal of Wuhan University, Wuhan, Vol. 35, No. 4, pp. 87-91, 2002.

[8] http://www.ngdc.noaa.gov/mgg/global/global.html.

[9] Porter M B, "The KRAKEN normal mode program", La Spezia: SACLANT Undersea Reserch Centre memorandum SM-245, 2001, pp. $1-202$.

[10] Weinberg H, Keenan R E, "Gaussian ray boundles for modeling highfrequency propagation loss under shallow-water conditions", J. Acoust. Soc. Am. , The Geology Society of America, Vol. 100, pp. 1421-1431, 1996.

[11] Zhang Xu, Zhang Yonggang, "Effects of sound speed profiles on convergence zone shift", Advance In Marine Science, Qingdao, Vol. 28, No. 3, pp. 311-317, 2010. 